\title{
Rapid Evaluation of Plant Extracts and Essential Oils for Antifungal Activity Against Botrytis cinerea
}

C. L. Wilson, Research Plant Pathologist, J. M. Solar, Chemist, A. El Ghaouth, Visiting Scientist, and M. E. Wisniewski, Plant Physiologist, USDA-ARS Appalachian Fruit Research Station, Kearneysville, WV 25430

\begin{abstract}
Wilson, C. L., Solar, J. M., El Ghaouth, A., and Wisniewski, M. E. 1997. Rapid evaluation of plant extracts and essential oils for antifungal activity against Botrytis cinerea. Plant Dis. 81:204-210.

A rapid assay to determine antifungal activity in plant extracts and essential oils is described. Wells in microtiter plates were loaded with Botrytis cinerea spores and plant extracts or essential oils. Subsequent changes in optical density following spore germination in the wells was measured after $24 \mathrm{~h}$ using an automatic microtiter plate reader driven by a software program developed for this purpose. Extracts from 345 plants and 49 essential oils were evaluated for their antifungal activity against $B$. cinerea. Among 345 plant extracts analyzed, 13 showed high levels of antifungal activity, with species of Allium and Capsicum predominating. Among the 49 essential oils tested, palmarosa (Cymbopogon martini), red thyme (Thymus zygis), cinnamon leaf (Cinnamomum zeylanicum), and clove buds (Eugenia caryophyllata) demonstrated the most antifungal activity against $B$. cinerea. The most frequently occurring constituents in essential oils showing high antifungal activity were: $D$-limonene, cineole; $\beta$-myrcene; $\alpha$-pinene, $\beta-$ pinene; and camphor.
\end{abstract}

Additional keywords: biocontrol, natural fungicides, volatiles

Among pesticides used to protect crops, fungicides were perceived until recently as relatively safe. A 1986 National Academy of Sciences (NAS) report (8) on pesticide residues on food indicated that fungicides pose more of a carcinogenic risk than insecticides and herbicides together. Therefore, synthetic fungicides are suspect in our food chain, and pressure is increasing to find safer alternatives. Additionally, resistance by pathogens to fungicides has rendered certain fungicides ineffective, creating a need for new ones with alternative modes of action. Present activities to find both natural and synthetic fungicides focus on finding compounds that are safe to humans and the environment.

Plant extracts $(4,5)$ and essential oils $(7,9)$ show antifungal activity against a wide range of fungi. Ark and Thompson (1) showed that garlic extracts contain a potent fungicide. They were able to effectively protect peaches against brown rot (Monilinia fructicola) with deodorized

Corresponding author: C. L. Wilson

E-mail: cwilson@asrr.arsusda.gov

Accepted for publication 18 November 1996.

Publication no. D-1997-0106-05R

This article is in the public domain and not copyrightable. It may be freely reprinted with customary crediting of the source. The American Phytopathological Society, 1997. garlic extract preparations. Singh et al. (9) found that essential oils from Cymbopogon martinii, C. oliveri, and Trachyspermum ammi exhibited strong antifungal activity against Helminthosporium oryzae.

Davidson and Parish (4) critically reviewed methods used for testing the efficacy of food antimicrobials. Most assays for antimicrobial compounds are labor intensive, time-consuming, and expensive. Microtiter plate methods have proved useful to the pharmaceutical and pesticide industries for rapid screening of bioactive compounds (3). We modified a microtiter plate assay to rapidly detect plant extracts and essential oils that may serve as alternatives to synthetic fungicides for the control of Botrytis cinerea.

\section{MATERIALS AND METHODS}

Plant extraction method. An extraction method was devised that is simple and does not require added chemicals. Fresh plant material was collected in resealable plastic bags and placed in a freezer for a minimum of $12 \mathrm{~h}$ at $-20^{\circ} \mathrm{C}$. As the plant material was tested, it was withdrawn from the freezer and allowed to thaw for a minimum of $20 \mathrm{~min}$ (Fig. 1D). Freezing and thawing fractured the plant cells and caused plant cell fluids to collect in the bag outside the tissue. The plastic bag was tilted so that the fluid collected in one corner. A small hole was cut at the corner, and extracted plant fluids were squeezed through the hole and collected in plastic weight boats (Fig. 1D). The extract was filter-sterilized by passing it through a .22$\mu \mathrm{m}$ syringe filter (Fig. 1E). A filtered 10\% extract solution was prepared by adding 50 $\mathrm{ml}$ of the sterile extract with $450 \mathrm{ml}$ of a $B$. cinerea spore suspension $\left(1 \times 10^{5}\right.$ conidia per $\mathrm{ml}$ ) in sterile malt extract broth.

Preparing the multi-well plates. Fifty $\mathrm{ml}$ of the plant extract and spore suspension mixture were pipetted into each well of a row of a 96 multi-well microtitration plate (Flow Laboratories) (Fig. 1F). A nontreated row of a spore suspension was added to each plate as a check, as well as a blank row. Background readings from these checks were subtracted by the software program from wells with fungal growth.

Each well was then sealed with a sheet of dental wax to prevent cross-contamination by volatiles (Fig. 1G). After 24-h incubation of the plates at $24^{\circ} \mathrm{C}$, the density of fungal growth in the wells was measured with an SLT Labinstrument Model

Table 1. Plant extracts with the highest persistent antifungal activity ${ }^{\mathrm{a}}$

\begin{tabular}{llll}
\hline Genus & Species & Common name & Family \\
\hline Adenocalyma & alleaceum & Garlic creeper & Bignoniaceae \\
Allium & ampeloprasum & Elephant garlic & Liliaceae \\
Allium & ramosum & Fragrant-flower garlic & Liliaceae \\
Allium & sativum & Serpent garlic & Liliaceae \\
Tulbaghia & violacea & Society garlic & Liliaceae \\
Capsicum & annuum & Pepper 'Chile' & Solanaceae \\
Capsicum & aпnuum & Pepper 'Jalapeno' & Solanaceae \\
Capsicum & annuum & Pepper 'Piquin' & Solanaceae \\
Capsicum & annuum & Pepper 'Sandia' & Solanaceae \\
Capsicum & annuum & Pepper 'Sweet' & Solanaceae \\
Capsicum & annum & Pepper 'Tepin' & Solanaceae \\
Capsicum & chinense & Pepper 'Habanero' & Solanaceae \\
Capsicum & frutescens & Pepper 'Tabasco' & Solanaceae \\
\hline
\end{tabular}

a Optical density reading under 40.00 OD. No Botrytis cinerea spore germination observed after $48 \mathrm{~h}$ at $10 \%$ dilution of the crude extract. 
EAR 400 AT microplate reader (Fig. 1A). This instrument was attached to a Wang computer system so that the absorbencyoptical density (492-mm filter) could be measured in each well and the difference analyzed.
Operation of microtiter plate reader. A software program was written in BASIC that allowed the operation of the microplate reader from a PC terminal. Once executed, the program checks the filter installed in the reader and displays the information at the computer terminal. At this point the reader is completely under software control. The program prompts the user for file names and indicates when plates should be changed. The user has the option for a single- or double-plate read-

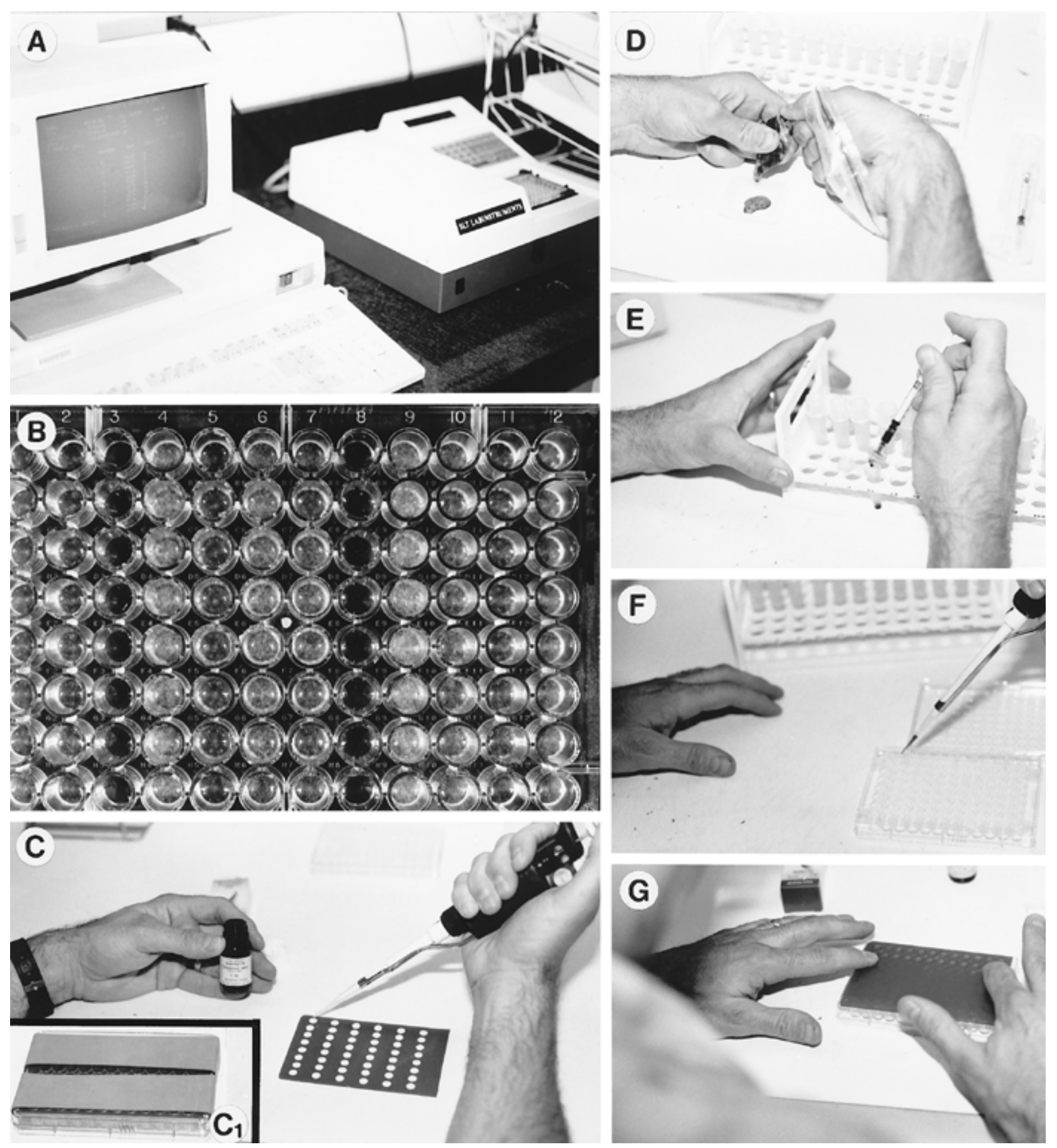

Fig. 1. Photographs of the assay of plant extracts and essential oils for antifungal activity. (A) Personal computer attached to an SLT Labinstrument Model EAR 400 AT microtiter plate reader. After plates containing extracts and Botrytis cinerea spores are incubated for $24 \mathrm{~h}$, they are placed in the tray of the computerdriven reader, which reads each individual well simultaneously for optical density and summarizes the results, which appear on the screen within 1 min. (B) Microtiter plate $48 \mathrm{~h}$ after $B$. cinerea spores and extracts have been incubated together at $20^{\circ} \mathrm{C}$. Row 3 is a blank with nothing added. Row 8 is a $10 \%$ dilution of a Capsicum extract. Row 9 is a spore suspension with no plant extract added. (C) Filter paper disks attached to a sheet of dental wax being loaded with essential oils. After the oils are loaded on the disk, the wax sheet is inverted over a microtiter plate and each well is sealed with slight pressure (Fig. 1G). $\left(\mathbf{C}_{\mathbf{1}}\right.$ insert) Microtiter plate sealed with wax, lid attached and held in place with two large rubber bands. (D) Plant material in resealable bag taken out of the freezer and allowed to thaw. Plant extract is being squeezed through hole cut in corner of bag. (E) Extract being filter sterilized. (F) Dilution of extract and spores being pipetted into wells of microtiter plate. (G) Dental wax being pressed over microtiter wells to seal individual wells. 
ing. The single-plate method was used in most instances. When the plant extract is pigmented, the double-plate method was used to distinguish between absorbency from the pigmentation and fungal growth. This method scans a blank plate first-a plate of extracted plant material without spores. The second plate reading contains the plant extract with spores. The software system subtracts the difference in optical density between plates. Both methods analyze 10 treatments with eight replicates and average the data for each treatment. Ten different extract-spore combinations are placed such that the eight well rows contain the same combination (Fig. 1B). The remaining two rows were used as controls, one with spores and no extract and the other a blank with nothing added. Once scanned, the data were placed in a data file.

In the screen, $10 \%$ dilutions of the plant extract were evaluated in the multi-well plates. Density readings ranged from optical density (OD) 470 to 12 on the multiplate reader. Density readings were made after $24 \mathrm{~h}$, and direct observations of spore germination were made with an in-

Table 2. Plant extracts with intermediate antifungal activity ${ }^{\mathrm{a}}$

\begin{tabular}{|c|c|c|c|}
\hline Genus & Species & Common name & Family \\
\hline Aglaia & odorata & Perfume plant & Meliaceae \\
\hline Allium & сера & Potato onion & Liliaceae \\
\hline Allium & fistolops & Welsh onion & Liliaceae \\
\hline Allium & schoenoprasum & Chive & Liliaceae \\
\hline Allium & tuberosum & Chinese chive & Liliaceae \\
\hline Capsicum & аппиит & 'Anaheim' & Solanaceae \\
\hline Capsicum & аппиит & 'Ancho' & Solanaceae \\
\hline Capsicum & аппиит & 'Big Jim’' & Solanaceae \\
\hline Capsicum & аппиит & 'Cayenne' & Solanaceae \\
\hline Capsicum & аппиит & 'Floral Gem' & Solanaceae \\
\hline Capsicum & annuum & 'Fresno Chile Grande' & Solanaceae \\
\hline Capsicum & аппиит & 'Holiday Time' & Solanaceae \\
\hline Capsicum & аппиит & 'Hungarian Yellow Wax' & Solanaceae \\
\hline Capsicum & аппиит & 'Large Cherry' & Solanaceae \\
\hline Capsicum & annuum & 'Mirasol' & Solanaceae \\
\hline Capsicum & аппиит & 'Sante Fe Grande' & Solanaceae \\
\hline Capsicum & аппиит & 'Serrano' & Solanaceae \\
\hline Capsicum & аппиит & 'Super Chile' & Solanaceae \\
\hline Capsicum & аппиит & 'Sweet Roumanian' & Solanaceae \\
\hline Capsicum & annuum & 'Yatsafusa' & Solanaceae \\
\hline Clematis & paniculata & Sweet autumn clematis & Ranunculaceae \\
\hline Hesperis & matronalis & Sweet Rocket & Cruciferae \\
\hline Isatis & tinctoria & Dyer's Woad & Cruciferae \\
\hline Juglans & nigra & Black walnut & Junglandaceae \\
\hline Liquidambar & styraciflua & Sweet gum & Hamamelidaceae \\
\hline Matricaria & sp. & 'Barths' & Compositae \\
\hline Oxalis & europaea & European yellow wood sorrel & Oxalidaceae \\
\hline Prunus & persica & Peach & Rosaceae \\
\hline Pyrus & communis & Pear & Rosaceae \\
\hline Satureja & acinos & Mother of thyme & Labiatae \\
\hline Taxus & canadensis & American yew & Taxaceae \\
\hline Taxus & media & Anglo-Japanese yew & Taxaceae \\
\hline
\end{tabular}

a Optical density reading under 40.00 OD and less than $10 \%$ Botrytis cinerea spore germination after $48 \mathrm{~h}$ with $10 \%$ dilution.

Table 3. Plant extracts with transitory antifungal activity ${ }^{\mathrm{a}}$

\begin{tabular}{|c|c|c|c|}
\hline Genus & Species & Common name & Family \\
\hline Achillea & millefolium & Yarrow & Compositae \\
\hline Amaranthus & cruentus & Amaranthus & Amaranthaceae \\
\hline Centranthus & ruber & Red valerian & Valerianaceae \\
\hline Chrysanthemum & parthenium & Chrysanthemum & Compositae \\
\hline Chrysanthemum & leucanthemum & Ox-eye daisy & Compositae \\
\hline Cistus & ladanifer & Laudanum & Cistaceae \\
\hline Cyperus & esculentus & Nutsedge & Cyperaceae \\
\hline Gaultheria & procumbens & Wintergreen & Ericaceae \\
\hline Glycyrrhiza & glabra & Licorice & Leguminosae \\
\hline Hedera & helix & English ivy & Araliaceae \\
\hline Linaria & vulgaris & Toadflax & Scrophlariaceae \\
\hline Pilea & pumila & Clearweed & Urticaceae \\
\hline Poterium & sanguisorba & Salad burnet & Rosaceae \\
\hline Prunella & vulgaris & Selfheal & Labiatae \\
\hline Senecio & obovatus & Squaw-weed & Compositae \\
\hline Vinca & minor & Periwinkle & Apocynaceae \\
\hline
\end{tabular}

a Optical density reading under $40.00 \mathrm{OD}$ and less than $50 \%$ spore germination after $12 \mathrm{~h}$ but more than $80 \%$ spore germination after $24 \mathrm{~h}$ with a $10 \%$ dilution. verted light microscope after 12 and $48 \mathrm{~h}$. A microscope field (40× objective) was chosen at random in each well of the plate, and the percent spore germination was recorded for each of eight wells and averaged.

Plant extracts were divided into four classes based on the OD of the fungal growth in the wells of the microtiter plates and the percent spore germination after 12 and $48 \mathrm{~h}$. The four classes were: (i) OD reading under 40.00 and no $B$. cinerea spore germination observed after $48 \mathrm{~h}$ at $10 \%$ dilution of the crude extract; (ii) OD reading under 40.00 and less than $10 \% B$. cinerea spore germination after $48 \mathrm{~h}$ with $10 \%$ dilution; (iii) OD reading under 40.00 and less than $50 \%$ spore germination after $12 \mathrm{~h}$ but more than $80 \%$ spore germination after $24 \mathrm{~h}$ with a $10 \%$ dilution; and (iv) OD reading of over 40.00 and more than $80 \%$ germination after $12 \mathrm{~h}$ with a $10 \%$ dilution. Table 1 represents those extracts described in class 1, Table 2 class 2, Table 3 class 3 , and Table 4 class 4 .

Evaluation of essential plant oils. Microtiter wells were inoculated with $B$. cinerea spores as described above. Forty-nine essential plant oils were obtained from Aroma Vera (P.O. Box 3609, Culver City, CA 90231). They were extracted by steam distillation or scarification by the manufacturer. To test the volatile fungicidal activity of the oils, $5 \mu \mathrm{l}$ was placed on small, 8-mm-diameter, filter paper disks attached to the dental wax seal for the multi-well plates (Fig. 1C).

In order to precisely align the filter paper disks, the wax seal was pressed lightly to the top of the multi-well plate so that an imprint of the top of the wells appeared on the wax. Individual filter paper disks were then placed in the center of each well imprint (Fig. 1C). After the essential oils were pipetted onto the disks, the wax seal was placed over the multi-well plate with the filter paper disk down. The wax seal was pressed tightly against the top of the plate to assure that each well was individually sealed. The lid of the plate was then attached, and two large rubber bands were used to hold the lid in place (Fig. 1C $\mathrm{C}_{1}$ ). Dilutions of the essential oils $(50,25,12.5$, $6.25,3.13,1.56,0.78$, and $0.39 \%$ ) were made with mineral oil, which showed no volatile fungicidal activity.

Spores were germinated at $24^{\circ} \mathrm{C}$ and observed after 24 and $40 \mathrm{~h}$. The results were recorded as spore germination $(+)$ or no spore germination $(-)$ (Table 4$)$.

Analysis of essential oil components. Chemical constituents of the essential oils were determined with a Hewlett Packard 5890 Series II Gas Chromatograph equipped with a Mass Selective Detector 5971 Series containing a fused silica capillary column, $12 \mathrm{~m} \times 0.02 \mathrm{~mm}$ i.d. $\times 0.33$ $\mu \mathrm{m}$ film thickness cross-linked 5\% phenyl methyl silicone (HP-1, Hewlett Packard, Wilmington, DE). Conditions employed 
Table 4. Volatile effect of plant essential oils on spore germination of Botrytis cinerea: (+) spore germination, (-) no spore germination

\begin{tabular}{|c|c|c|c|c|c|c|c|c|c|}
\hline \multirow[b]{2}{*}{ Essential oil } & \multicolumn{9}{|c|}{ Concentration (\%) } \\
\hline & 100 & 50 & 25 & 12.5 & 6.25 & 3.13 & 1.56 & 0.78 & 0.39 \\
\hline \multicolumn{10}{|l|}{ Basil } \\
\hline \multicolumn{10}{|c|}{ (Ocimum basilicum) } \\
\hline $40 \mathrm{~h}$ & + & & & & & & & & \\
\hline \multicolumn{10}{|l|}{ Bergamot } \\
\hline \multicolumn{10}{|c|}{ (Citrus bergamia) } \\
\hline $24 \mathrm{~h}$ & + & & & & & & & & \\
\hline $40 \mathrm{~h}$ & + & & & & & & & & \\
\hline \multicolumn{10}{|l|}{ Birch } \\
\hline \multicolumn{10}{|l|}{ (Betula nigra) } \\
\hline $24 \mathrm{~h}$ & - & - & - & - & + & & & & \\
\hline $40 \mathrm{~h}$ & - & - & - & + & + & & & & \\
\hline Carrot seed & & & & & & & & & \\
\hline (Daucus carot & & & & & & & & & \\
\hline $24 \mathrm{~h}$ & - & - & - & + & + & & & & \\
\hline $40 \mathrm{~h}$ & - & + & + & + & + & & & & \\
\hline Cedar wood & & & & & & & & & \\
\hline (Juniperus me & & & & & & & & & \\
\hline $24 \mathrm{~h}$ & + & & & & & & & & \\
\hline $40 \mathrm{~h}$ & + & & & & & & & & \\
\hline Chamomile $\mathrm{m}$ & & & & & & & & & \\
\hline (Anthemis mix & & & & & & & & & \\
\hline $24 \mathrm{~h}$ & - & - & + & + & + & & & & \\
\hline $40 \mathrm{~h}$ & - & + & + & + & + & & & & \\
\hline Cinnamon lea & & & & & & & & & \\
\hline (Cinnamomun & & & & & & & & & \\
\hline $24 \mathrm{~h}$ & - & - & - & - & - & - & - & + & + \\
\hline $40 \mathrm{~h}$ & - & - & - & - & - & - & - & + & + \\
\hline Citronella & & & & & & & & & \\
\hline (Cymbopogon & & & & & & & & & \\
\hline $24 \mathrm{~h}$ & - & - & - & - & - & + & & & \\
\hline $40 \mathrm{~h}$ & - & - & - & - & - & + & & & \\
\hline Clary sage & & & & & & & & & \\
\hline (Salvia sclarec & & & & & & & & & \\
\hline $24 \mathrm{~h}$ & - & + & + & + & + & & & & \\
\hline $40 \mathrm{~h}$ & + & + & + & + & + & & & & \\
\hline Clove buds & & & & & & & & & \\
\hline (Eugenia cary & & & & & & & & & \\
\hline $24 \mathrm{~h}$ & - & - & - & - & - & - & - & - & + \\
\hline $40 \mathrm{~h}$ & - & - & - & - & - & - & - & + & + \\
\hline Cypress & & & & & & & & & \\
\hline (Cupressus se & & & & & & & & & \\
\hline $24 \mathrm{~h}$ & - & + & + & + & + & & & & \\
\hline $40 \mathrm{~h}$ & + & + & + & + & + & & & & \\
\hline Elemi & & & & & & & & & \\
\hline (Canarium luz & & & & & & & & & \\
\hline $24 \mathrm{~h}$ & + & & & & & & & & \\
\hline $40 \mathrm{~h}$ & + & & & & & & & & \\
\hline Eucalyptus & & & & & & & & & \\
\hline (Eucalyptus al & & & & & & & & & \\
\hline $24 \mathrm{~h}$ & - & + & + & + & + & & & & \\
\hline $40 \mathrm{~h}$ & + & + & + & + & + & & & & \\
\hline Fennel & & & & & & & & & \\
\hline (Foeniculum $\mathrm{v}$ & & & & & & & & & \\
\hline $24 \mathrm{~h}$ & + & & & & & & & & \\
\hline $40 \mathrm{~h}$ & + & & & & & & & & \\
\hline $\begin{array}{l}\text { Fir } \\
\text { (Abies balsam }\end{array}$ & & & & & & & & & \\
\hline $24 \mathrm{~h}$ & - & + & + & + & + & & & & \\
\hline $40 \mathrm{~h}$ & + & + & + & + & + & & & & \\
\hline Geranium & & & & & & & & & \\
\hline (Pelargonium & & & & & & & & & \\
\hline $24 \mathrm{~h}$ & - & - & - & - & + & & & & \\
\hline $40 \mathrm{~h}$ & - & - & - & + & + & & & & \\
\hline Grapefruit & & & & & & & & & \\
\hline (Citrus paradi & & & & & & & & & \\
\hline $24 \mathrm{~h}$ & + & & & & & & & & \\
\hline $40 \mathrm{~h}$ & + & & & & & & & & \\
\hline Juniper & & & & & & & & & \\
\hline (Juniperus cor & & & & & & & & & \\
\hline $24 \mathrm{~h}$ & + & & & & & & & & \\
\hline $40 \mathrm{~h}$ & + & & & & & & & & \\
\hline & & & & & & & & ontin & tge) \\
\hline
\end{tabular}


Table 4. (continued from preceding page)

\begin{tabular}{|c|c|c|c|c|c|c|c|c|c|}
\hline \multirow[b]{2}{*}{ Essential oil } & \multicolumn{9}{|c|}{ Concentration $(\%)$} \\
\hline & 100 & 50 & 25 & 12.5 & 6.25 & 3.13 & 1.56 & 0.78 & 0.39 \\
\hline \multicolumn{10}{|c|}{$\begin{array}{l}\text { Lavandin } \\
\text { (Lavandula fragrans) }\end{array}$} \\
\hline $24 \mathrm{~h}$ & - & - & - & + & + & & & & \\
\hline $40 \mathrm{~h}$ & - & - & + & + & + & & & & \\
\hline \multirow{2}{*}{\multicolumn{10}{|c|}{$\begin{array}{l}\text { Lavender } \\
\text { (Lavandula officinalis) }\end{array}$}} \\
\hline & & & & & & & & & \\
\hline $24 \mathrm{~h}$ & - & - & - & + & + & & & & \\
\hline $40 \mathrm{~h}$ & - & + & + & + & + & & & & \\
\hline \multicolumn{10}{|l|}{ Lemon } \\
\hline \multicolumn{10}{|c|}{ (Citrus limonum) } \\
\hline $24 \mathrm{~h}$ & + & & & & & & & & \\
\hline $40 \mathrm{~h}$ & + & & & & & & & & \\
\hline \multicolumn{10}{|l|}{ Lemongrass } \\
\hline (Cymbopogon & & & & & & & & & \\
\hline $24 \mathrm{~h}$ & - & - & - & - & - & & & & \\
\hline $40 \mathrm{~h}$ & - & - & - & - & + & & & & \\
\hline Lime & & & & & & & & & \\
\hline (Citrus limettc & & & & & & & & & \\
\hline $24 \mathrm{~h}$ & + & & & & & & & & \\
\hline $40 \mathrm{~h}$ & + & & & & & & & & \\
\hline Litsea & & & & & & & & & \\
\hline (Litsea cubebc & & & & & & & & & \\
\hline $24 \mathrm{~h}$ & - & - & - & - & - & + & + & & \\
\hline $40 \mathrm{~h}$ & - & - & - & - & - & + & + & & \\
\hline Marjoram & & & & & & & & & \\
\hline (Thymus mast & & & & & & & & & \\
\hline $24 \mathrm{~h}$ & - & - & - & + & + & & & & \\
\hline $40 \mathrm{~h}$ & - & - & + & + & + & & & & \\
\hline Mugwort & & & & & & & & & \\
\hline (Artemisia vul & & & & & & & & & \\
\hline $24 \mathrm{~h}$ & - & - & - & + & + & & & & \\
\hline $40 \mathrm{~h}$ & - & - & + & + & + & & & & \\
\hline Myrtle & & & & & & & & & \\
\hline (Myrtus comm & & & & & & & & & \\
\hline $24 \mathrm{~h}$ & - & - & + & + & + & & & & \\
\hline $40 \mathrm{~h}$ & - & + & + & + & + & & & & \\
\hline Niaouli & & & & & & & & & \\
\hline (Melaleuca vi & & & & & & & & & \\
\hline $24 \mathrm{~h}$ & - & - & - & + & + & & & & \\
\hline $40 \mathrm{~h}$ & - & + & + & + & + & & & & \\
\hline Nutmeg & & & & & & & & & \\
\hline (Myristica fra & & & & & & & & & \\
\hline $24 \mathrm{~h}$ & - & + & + & + & + & & & & \\
\hline $40 \mathrm{~h}$ & + & + & + & + & + & & & & \\
\hline Orange & & & & & & & & & \\
\hline (Citrus auran & & & & & & & & & \\
\hline $24 \mathrm{~h}$ & + & & & & & & & & \\
\hline $40 \mathrm{~h}$ & + & & & & & & & & \\
\hline Oregano & & & & & & & & & \\
\hline (Coridothymu & & & & & & & & & \\
\hline $24 \mathrm{~h}$ & - & - & - & - & - & - & + & & \\
\hline $40 \mathrm{~h}$ & - & - & - & - & - & + & + & & \\
\hline Palmarosa & & & & & & & & & \\
\hline (Cymbopogon & & & & & & & & & \\
\hline $24 \mathrm{~h}$ & - & - & - & - & - & - & - & - & + \\
\hline $40 \mathrm{~h}$ & - & - & - & - & - & - & - & - & + \\
\hline Patchouly & & & & & & & & & \\
\hline (Pogostemon & & & & & & & & & \\
\hline $24 \mathrm{~h}$ & + & & & & & & & & \\
\hline $40 \mathrm{~h}$ & + & & & & & & & & \\
\hline Peppermint & & & & & & & & & \\
\hline (Mentha piper & & & & & & & & & \\
\hline $24 \mathrm{~h}$ & - & - & - & - & + & & & & \\
\hline $40 \mathrm{~h}$ & - & - & + & + & + & & & & \\
\hline Petitgrain & & & & & & & & & \\
\hline (Citrus auran & & & & & & & & & \\
\hline $24 \mathrm{~h}$ & - & - & - & + & + & & & & \\
\hline $40 \mathrm{~h}$ & - & + & + & + & + & & & & \\
\hline Pine & & & & & & & & & \\
\hline (Pinus sylvest & & & & & & & & & \\
\hline $24 \mathrm{~h}$ & - & + & + & + & + & & & & \\
\hline $40 \mathrm{~h}$ & + & + & + & + & + & & & & \\
\hline & & & & & & & & (contin & ext page) \\
\hline
\end{tabular}


Table 4. (continued from preceding page)

\begin{tabular}{|c|c|c|c|c|c|c|c|c|c|}
\hline \multirow[b]{2}{*}{ Essential oil } & \multicolumn{9}{|c|}{ Concentration (\%) } \\
\hline & 100 & $\mathbf{5 0}$ & 25 & 12.5 & 6.25 & 3.13 & 1.56 & 0.78 & 0.39 \\
\hline \multicolumn{10}{|c|}{ Rosemary } \\
\hline \multicolumn{10}{|c|}{ (Rosmarinus officinalis) } \\
\hline $24 \mathrm{~h}$ & _- & _- & + & + & & & & & \\
\hline $40 \mathrm{~h}$ & - & + & + & + & + & & & & \\
\hline \multicolumn{10}{|l|}{ Rosewood } \\
\hline \multicolumn{10}{|c|}{ (Aniba roseaodora) } \\
\hline $24 \mathrm{~h}$ & - & - & - & - & - & & & & \\
\hline $40 \mathrm{~h}$ & - & - & - & - & + & & & & \\
\hline \multicolumn{10}{|c|}{$\begin{array}{l}\text { Sage lavandulifolia } \\
\text { (Salvia officinalis) }\end{array}$} \\
\hline $24 \mathrm{~h}$ & - & - & - & + & + & & & & \\
\hline $40 \mathrm{~h}$ & - & - & + & + & + & & & & \\
\hline \multicolumn{10}{|l|}{ Savory } \\
\hline \multicolumn{10}{|c|}{ (Satureja montana) } \\
\hline $24 \mathrm{~h}$ & _- & _- & _- & _- & - & & & & \\
\hline $40 \mathrm{~h}$ & - & - & - & - & + & & & & \\
\hline \multicolumn{10}{|l|}{ Peppermint } \\
\hline \multicolumn{10}{|c|}{ (Mentha poperita) } \\
\hline $24 \mathrm{~h}$ & - & - & - & - & - & - & + & + & + \\
\hline $40 \mathrm{~h}$ & - & - & - & - & + & + & + & + & + \\
\hline \multicolumn{10}{|l|}{ Spike } \\
\hline \multicolumn{10}{|c|}{ (Lavandula spica) } \\
\hline $24 \mathrm{~h}$ & - & - & - & + & + & & & & \\
\hline $40 \mathrm{~h}$ & - & _- & - & + & + & & & & \\
\hline \multicolumn{10}{|l|}{ Spruce } \\
\hline \multicolumn{10}{|c|}{ (Picea mariana) } \\
\hline $24 \mathrm{~h}$ & + & & & & & & & & \\
\hline $40 \mathrm{~h}$ & + & & & & & & & & \\
\hline \multicolumn{10}{|l|}{ Tangerine } \\
\hline \multicolumn{10}{|c|}{ (Citrus reticulata) } \\
\hline $24 \mathrm{~h}$ & + & & & & & & & & \\
\hline $40 \mathrm{~h}$ & + & & & & & & & & \\
\hline \multicolumn{10}{|l|}{ Tarragon } \\
\hline (Artemisia $d r$ & & & & & & & & & \\
\hline $24 \mathrm{~h}$ & + & & & & & & & & \\
\hline $40 \mathrm{~h}$ & + & & & & & & & & \\
\hline Tea tree & & & & & & & & & \\
\hline (Melaleuca al & & & & & & & & & \\
\hline $24 \mathrm{~h}$ & - & _- & _- & - & + & & & & \\
\hline $40 \mathrm{~h}$ & - & - & - & + & + & & & & \\
\hline Thyme lemon & & & & & & & & & \\
\hline (Thymus hiem & & & & & & & & & \\
\hline $24 \mathrm{~h}$ & - & - & + & + & + & & & & \\
\hline $40 \mathrm{~h}$ & - & + & + & + & + & & & & \\
\hline Thyme red & & & & & & & & & \\
\hline (Thymus zygis & & & & & & & & & \\
\hline $24 \mathrm{~h}$ & - & - & - & - & - & - & - & - & + \\
\hline $40 \mathrm{~h}$ & - & - & - & - & - & - & - & - & + \\
\hline Vetiver & & & & & & & & & \\
\hline (Andropogon & & & & & & & & & \\
\hline $24 \mathrm{~h}$ & - & + & + & + & + & & & & \\
\hline $40 \mathrm{~h}$ & + & + & + & + & + & & & & \\
\hline
\end{tabular}

were the following: carrier gas, ultra high purity helium (GM Industries, Hagerstown, $\mathrm{MD)}$ at 15 psig. Oven temperature program: $45^{\circ} \mathrm{C}(3 \mathrm{~min})$ to $300^{\circ} \mathrm{C}(1 \mathrm{~min})$ at $10^{\circ} \mathrm{C} \mathrm{min}^{-1}$.

The "as received" essential oils were diluted approximately 200-fold (vol/ vol) in HPLC grade methanol (Mallinckrodt Specialty Chemicals, Paris, KY) and injected into the Chromatograph in 1.0- $\mu$ l aliquots. The major constituent(s) was identified with the aid of a computer-driven algorithm to eliminate possibilities and then by matching the mass spectrum of the analyte with that of a library of electron impact mass spectra.

\section{RESULTS}

Plant extracts. Plant extracts showing the greatest antifungal activity were those from species of Allium and Capsicum (Table 1). A 10\% dilution of these extracts registered under 40.00 OD in fungal growth and completely inhibited spore germination of $B$. cinerea after 24 and $48 \mathrm{~h}$.

Another group of plant extracts registered under 40.00 OD in fungal growth and under $10 \%$ B. cinerea spore germination with a $10 \%$ dilution (Table 2). This group was more diverse than those in Table 1 but nevertheless contained predominately species of Allium and Capsicum.

A more diverse group of plants than those in Tables 1 and 2 (Table 3) registered under 40.00 OD and showed some transitory fungicidal activity with less than $50 \%$ spore germination after $12 \mathrm{~h}$ but more than $80 \%$ germination after $24 \mathrm{~h}$ with a $10 \%$ dilution.

Those plant extracts (283 in number) that registered over 40.00 OD on the primary screen with the microtiter plate reader and had more than $80 \%$ germination after $12 \mathrm{~h}$ with a $10 \%$ dilution of the extract were considered to have low or no antifungal activity (data not shown).

Essential oils. Palmarosa (Cymbopogon martini) and red thyme (Thymus zygis) had essential oils that showed the greatest inhibition of spore germination of $B$. cinerea at 
Table 5. Most frequently occurring constituents in the essential oils tested and the plants in which they occurred

\begin{tabular}{|c|c|c|}
\hline Constituent & Plant & $\begin{array}{c}\text { Retention } \\
\text { time }\end{array}$ \\
\hline$D$-Limonene & $\begin{array}{l}\text { Abies balsamea } \\
\text { Artemisia vulgaris } \\
\text { Citrus aurantium } \\
\text { Citrus bergamia } \\
\text { Citrus limetta } \\
\text { Citrus limonum } \\
\text { Citrus paradisi } \\
\text { Citrus reticulata } \\
\text { Coridothymus capital } \\
\text { Cupressus sempervirens } \\
\text { Cymbopogon nardus } \\
\text { Cymbopogon citratus } \\
\text { Litsea cubeba } \\
\text { Ocimum basilicum } \\
\text { Pelargonium roseum } \\
\text { Pinus sylvestris } \\
\text { Rosmarinus officinalis } \\
\text { Thymus hiemalis } \\
\text { Thymus zygis }\end{array}$ & $\begin{array}{l}7.72 \\
7.72 \\
7.74 \\
7.70 \\
7.71 \\
7.81 \\
7.73 \\
7.70 \\
7.70 \\
7.73 \\
7.75 \\
7.74 \\
7.70 \\
7.71 \\
7.74 \\
7.74 \\
7.72 \\
7.70 \\
7.75\end{array}$ \\
\hline Cineole & $\begin{array}{l}\text { Artemisia vulgaris } \\
\text { Coridothymus capital } \\
\text { Eucalyptus austalis } \\
\text { Lavandula fragrans } \\
\text { Lavandula spica } \\
\text { Melaleuca alternifolia } \\
\text { Myrtus communis } \\
\text { Ocimum basilicum } \\
\text { Rosmarinus officinalis } \\
\text { Salvia officinalis } \\
\text { Thymus hiemalis } \\
\text { Thymus masticinia }\end{array}$ & $\begin{array}{l}7.72 \\
7.70 \\
7.79 \\
7.71 \\
7.78 \\
7.71 \\
7.74 \\
7.70 \\
7.71 \\
7.72 \\
7.70 \\
7.75\end{array}$ \\
\hline$\beta$-Myrcene & $\begin{array}{l}\text { Aniba roseaodora } \\
\text { Citrus aurantium } \\
\text { Cymbopogon martini } \\
\text { Cymbopogon nardus } \\
\text { Lavandula officinalis } \\
\text { Pelargonium roseum }\end{array}$ & $\begin{array}{l}7.71 \\
7.71 \\
7.23 \\
7.21 \\
7.21 \\
7.21 \\
7\end{array}$ \\
\hline$\beta$-Pinene & $\begin{array}{l}\text { Citrus limonum } \\
\text { Pinus sylvestris }\end{array}$ & $\begin{array}{l}6.99 \\
7.00\end{array}$ \\
\hline$\alpha$-Pinene & $\begin{array}{l}\text { Cupressus sempervirens } \\
\text { Pinus sylvestris }\end{array}$ & $\begin{array}{l}6.38 \\
6.40\end{array}$ \\
\hline Camphor & $\begin{array}{l}\text { Artemisia vulgaris } \\
\text { Lavandula spica } \\
\text { Salvia lavandulifolia } \\
\text { Thymus hiemalis }\end{array}$ & $\begin{array}{l}9.07 \\
9.04 \\
9.05 \\
9.02\end{array}$ \\
\hline
\end{tabular}

the lowest concentration (Table 4). Spore germination was completely inhibited at a $0.78 \%$ dilution after $40 \mathrm{~h}$. The next best inhibitor of spore germination was clove buds (Eugenia caryophyllata), which showed complete inhibition of spore germination at a $0.78 \%$ dilution up to $24 \mathrm{~h}$ (Table 4). Another essential oil with notable activity was cinnamon leaf (Cinnamomum zeylanicum), which inhibited spore germination at a $1.56 \%$ dilution after $40 \mathrm{~h}$. The relative activity of the other volatile oils tested can be found in Table 4 .
Essential oil components. The chemical constituents in the essential oils derived by a methanol extraction are presented in Table 5. We are in the process of determining the fungicidal activity of the individual components singly and in combination.

\section{DISCUSSION}

Within the large reservoir of natural fungicides that exist in plants and microorganisms, it is reasonable that examples exist that would serve as safe and effective alternatives to synthetic fungicides. Such compounds, if properly formulated and applied, could be used directly or could serve as templates for synthetic analogs. Microbially derived natural compounds have also been proposed as alternatives to synthetic fungicides. Antibiotics such as iturin, produced by Bacillus subtilis (6), are effective as fungicides against a number of plant pathogens. However, there is considerable resistance to the use of antibiotics in agriculture. It has been argued that such use will risk the development of resistance in animal pathogens to the antibiotic and thereby diminishes its usefulness in animal disease therapy.

The most plants showing antifungal activity among the 345 plant extracts tested were species of Allium or Capsicum. Although Allium sativum was demonstrated early to have good antifungal activity and to be useful as a postharvest treatment (1), it has not been commercialized. Essential oils have long been recognized as having good fungitoxic compounds (9), but they have not been developed into products for postharvest treatments, since industry finds it easier to patent and protect newly synthesized compounds than natural plant products.

Latent infections are especially difficult to control in harvested commodities, because the pathogen resides in an inactive state within the host tissue. Nonsystemic, synthetic fungicides and biological control agents are ineffective in controlling such infections (10). Natural fungicidal volatiles may be useful in controlling latent infections. Wilson et al. (11) found that a number of the volatiles that emanate from peaches as they ripen are fungicidal. It is possible to fumigate peaches with one of these volatiles (benzyaldehyde) and protect them against decay (C. L. Wilson, unpublished). Perhaps volatile fungitoxic compounds from plant essential oils could be utilized to control postharvest diseases of fruits and vegetables. With the precipitous withdrawal of methyl bromide as a fumigant, it may be profitable to explore natural plant volatiles as alternatives.

Natural plant-derived fungicides should provide a wide variety of compounds as alternatives to synthetic fungicides, both as fumigants and as contact pesticides (2). They may also prove valuable as "lead structures" for the development of synthetic compounds. It behooves us to explore more intensely this rich source of fungicides.

\section{ACKNOWLEDGMENTS}

We thank Brian E. Otto for his excellent technical assistance and Gary Lightner for writing the software program.

\section{LITERATURE CITED}

1. Ark, P. A., and Thompson, J. P. 1959. Control of certain diseases of plants with antibiotics from garlic (Allium sativum L.). Plant Dis. Rep. 43:276-282.

2. Cutler, H. G., and Hill, R. A. 1994. Natural fungicides and their delivery systems as alternatives to synthetics. Pages 135-152 in: Biological Control of Postharvest Diseases - Theory and Practice. C. L. Wilson and M. E. Wisniewski, eds. CRC Press, Boca Raton, FL.

3. Daeschel, M. A. 1992. Procedures to detect antimicrobial activities of microorganisms. Pages 57-80 in: Food Biopreservatives of Microbial Origin. B. Ray and M. Daeschel, eds. CRC Press, Boca Raton, FL.

4. Davidson, P. M., and Parish, M. E. 1989 Methods for testing the efficacy of food antimicrobials. Food Technol. 43:148-155.

5. Grange, M., and Ahmed, S. 1988. Handbook of Plants with Pest Control Properties. John Wiley \& Sons, New York

6. Gueldner, R. C., Reilly, C. C., Pusey, P. L., Costello, C. E., and Arrendale, R. F. 1988 Isolation and identification of iturins as antifungal peptides in biological control of peach brown rot with Bacillus subtilis. J. Agric. Food Chem. 36:366-370.

7. Kurita, N., Makoto, M., Kurane, R., and Takahara, Y. 1981. Antifungal activity of components of essential oils. Agric. Biol. Chem. 45:945-952.

8. Research Council, Board of Agriculture 1987. Regulating Pesticides in Food - The Delaney Paradox. National Academy Press, Washington, DC.

9. Singh, A. K., Dickshit, A., Sharma, M. L. and Dixit, S. N. 1980. Fungitoxic activity of some essential oils. Econ. Bot. 34:186 190.

10. Wilson, C. L., and El Ghaouth, A. 1993. Multifaceted biological control of postharvest diseases of fruits and vegetables. Pages 181185 in: Pest Management: Biologically Based Technologies. R. D. Lumsden and J. L. Vaughn, eds. American Chemical Society, Washington, DC

11. Wilson, C. L., Franklin, J. D., and Otto, B. E 1987. Fruit volatiles inhibitory to Monilinia fructicola and Botrytis cinerea. Plant Dis. 71:316-319. 